\title{
Canonical Sequent Proofs via Multi-Focusing
}

\author{
Kaustuv Chaudhuri ${ }^{1}$, Dale Miller ${ }^{2}$, and Alexis Saurin ${ }^{3}$ \\ 1 INRIA Saclay - Île-de-France, Kaustuv .Chaudhuri@inria.fr \\ 2 INRIA Saclay - Île-de-France \& LIX, École Polytechnique, Dale.Miller@inria.fr \\ ${ }^{3}$ INRIA Saclay - Île-de-France \& LIX, École Polytechnique, Alexis.Saurin@inria.fr
}

\begin{abstract}
The sequent calculus admits many proofs of the same conclusion that differ only by trivial permutations of inference rules. In order to eliminate this "bureaucracy" from sequent proofs, deductive formalisms such as proof nets or natural deduction are usually used instead of the sequent calculus, for they identify proofs more abstractly and geometrically. In this paper we recover permutative canonicity directly in the cut-free sequent calculus by generalizing focused sequent proofs to admit multiple foci, and then considering the restricted class of maximally multifocused proofs. We validate this definition by proving a bijection to the well-known proof-nets for the unit-free multiplicative linear logic, and discuss the possibility of a similar correspondence for larger fragments.
\end{abstract}

\section{Introduction}

Sequent calculus proofs are much less proof objects than they are traces of the computation of a more abstract proof object. In particular, the infernece rules of the sequent calculus are minute and there are many choices in the order of their application that seem equivalent although, formally, they result in different sequent proofs. One way to get a more abstract notion of proof is to declare that two cut-free proofs are equivalent if it is possible to permute the inference rules in one to get the other. Such equivalence classes are unsatisfactory for at least two reasons. First, computing permutations of inference rules might require examining and reorganizing arbitrary parts of a proof: attempting to move a given inference rule to the bottom of a proof could cause changes to many parts of the proof. Second, since equivalence classes are not, themselves, inductive structures, familiar arguments involving inductive reasoning over proof structures cannot be applied easily to equivalence classes. Many people working in proof theory and particularly those interested in the problem of the identity of proofs discard sequent proofs for more abstract proof structures like natural deduction proofs or proof nets. In these later objects, a more geometric structure of proofs requires less sequentialization of inference rules and allows one to work on proofs more abstractly.

We shall argue in this paper that one does not need to discard the sequent calculus in order to factor out many of these irrelevant sequentializations of inference rules. We shall show that there are, in fact, normal forms of sequent proofs that provide unique representatives of their permutative equivalence classes. To be concrete, we shall assume a setting of the standard cut-free sequent calculus for multiplicative-additive linear logic (MALL), including units and literals. Motivating the construction of canonical representatives is as follows. A first step is to consider only focused proofs [2],

Please use the following format when citing this chapter:

Chaudhuri, K., Miller, D. and Saurin, A., 2008, in IFIP International Federation for Information Processing, Volume 273; Fifth IFIP International Conference on Theoretical Computer Science; Giorgio Ausiello, Juhani Karhumäki, Giancarlo Mauri, Luke Ong; (Boston: Springer), pp. 383-396. 
with a strict alternation of negative (invertible) and positive (focused) phases. Focused proofs systems can be used to distinguish between micro rules, i.e., introduction rules in the ordinary sequent calculus, and the macro rules that comprise an entire focusing phases and correspond to the introduction of synthetic connectives [5]. A first abstraction is then to consider proofs as built up from macro rules introducing synthetic connectives. Unfortunately, this layer of abstraction does not yield canonical representatives of equivalence classes since the selection of foci is still sequentialized even when the selection order is irrelevant. Such parallelism can be captured by the addition of the multi-focus rule that permits focusing on several formulas within one phase. If we then require that such multi-focus inference rules select a "maximal focus" then, as we show in Section 4, we have achieved canonical representatives of equivalence classes of proofs.

Proof nets for MLL and MALL have been used also as abstractions of the class of cut-free proofs under the equivalence of permuting inference rules. We show that maximally multi-focused sequent proofs (modulo the weak "iso-polar" equivalence) are in one-to-one correspondence with MLL proof nets [9]: we show how to uniquely associate a maximally multi-focused proof to an MLL proof net. We also discuss proof nets in MALL without units $[10,12]$ and for other fragments of linear logic: maximal multi-focusing proofs should also be applicable in various other richer logics where the nature of proof nets is less well developed or satisfying, such as linear logic with units and exponentials.

This paper is organized as follows: in Sec. 2 we recall the sequent calculus for MALL. In Sec. 3 we present our multi-focal generalization of Andreoli's focusing calculus. In Sec. 4 we define the notion of maximality and prove the key canonicity result (Theorem 7). In Sec. 5 we exhibit a one-to-one correspondence between maximally multi-focused proofs and proof-nets for MLL without units.

\section{Sequent calculus for MALL}

MALL formulas are defined by the following grammar:

$$
A, B, \ldots::=a\left|a^{\perp}\right| A \otimes B|\mathbf{1}| A \not B B|\perp| A \& B|\top| A \oplus B \mid \mathbf{0}
$$

A literal is either an atomic formula, written using minuscule scheme variables $(a, b, \ldots)$, or it is a negated atom $\left(a^{\perp}, b^{\perp}, \ldots\right)$. As usual, MALL formulas are assumed to be in negation-normal form, and the pairs $(\otimes, \not{X}),(\mathbf{1}, \perp),(\&, \oplus)$, and $(\top, \mathbf{0})$ are de Morgan duals, i.e., $(A \otimes B)^{\perp}=A^{\perp} \ngtr B^{\perp}$, etc. The sequent calculus for MALL uses one-sided sequents of the form $\vdash \Gamma$, where the context $\Gamma$ is a multiset of formulas. Figure 1 contains the standard proof rules for such sequents [9].

Script majuscule letters $\mathcal{D}, \mathcal{E}, \ldots$ are used to denote proofs and the expression $\mathcal{D} \vdash \Gamma$ signifies that $\mathcal{D}$ is a proof of $\vdash \Gamma$. It is well-known that the following cut and (nonatomic) initial rules are admissible. 


$$
\begin{aligned}
& \frac{}{\vdash a, a^{\perp}} \mathrm{I} \quad \frac{\vdash \Gamma, A \quad \vdash \Delta, B}{\vdash \Gamma, \Delta, A \otimes B} \otimes \quad \overline{\vdash 1} \mathbf{1} \frac{\vdash \Gamma, A, B}{\vdash \Gamma, A \ngtr B} \ngtr \quad \frac{\vdash \Gamma}{\vdash \Gamma, \perp} \perp \\
& \frac{\vdash \Gamma, A \quad \vdash \Gamma, B}{\vdash \Gamma, A \& B} \& \quad \frac{\vdash \Gamma, A_{i}}{\vdash \Gamma, \top} \top \quad \frac{\vdash \Gamma, A_{1} \oplus A_{2}}{\oplus_{i}}
\end{aligned}
$$

Fig. 1 Sequent calculus for MALL. In the $\oplus_{i}$ rule, $i \in\{1,2\}$.

$$
\frac{\vdash \Gamma, A \quad \vdash \Delta, A^{\perp}}{\vdash \Gamma, \Delta} \mathrm{C} \quad \text { and } \quad \overline{\vdash A, A^{\perp}} \mathrm{I}^{*}
$$

Local permutations of inference rules form a natural relation between cut-free proofs [13]. For example, in a proof of the form

$$
\frac{\mathcal{D} \vdash \Gamma, A \quad \frac{\mathcal{E} \vdash \Delta, B, C \quad \mathcal{F} \vdash \Delta, B, D}{\vdash \Delta, B, C \& D} \&,}{\vdash \Gamma, \Delta, A \otimes B, C \& D} \otimes
$$

the order of the $\otimes$ and $\&$ rules may be locally switched to yield the proof

$$
\frac{\mathcal{D} \vdash \Gamma, A \quad \mathcal{E} \vdash \Delta, B, C}{\frac{\vdash \Gamma, \Delta, A \otimes B, C}{\vdash \Gamma, \Delta, A \otimes B, C \& D} \frac{\mathcal{D} \vdash \Gamma, A \quad \mathcal{F} \vdash \Delta, B, D}{\vdash \Gamma, \Delta, A \otimes B, D} \otimes} \otimes
$$

This switching causes the proof $\mathcal{D}$ to be duplicated in (2), but does not alter the constituent sub-proofs $\mathcal{D}, \mathcal{E}$ and $\mathcal{F}$. We denote a site of a local permutation, i.e., a pair of neighbouring inference rules $r_{1}$ followed by $r_{2}$ as $r_{1} / r_{2}$; for example, (1) ends with a $\& / \otimes$ along the right branch of the final rule.

Consider, instead, the following proof figures.

$$
\frac{\mathcal{D} \vdash \Gamma, A \quad \overline{\vdash \Delta, B, \top}}{\vdash \Gamma, \Delta, A \otimes B, \top} \otimes \frac{}{\vdash \Gamma, \Delta, A \otimes B, \top}{ }^{\top}
$$

Moving from left-to-right can be seen as moving the $T$ inference rule below the $\otimes$ rule: in the process the entire proof $\mathcal{D}$ is deleted. Since we wish to establish an equivalence based on permutations, moving from right-to-left can be seen as "creating" the proof $\mathcal{D}$. While deletion of proofs can be seen as problematic when one is attempting to capture the "essence" of proofs, creation is certainly problematic in this sense. Thus, we introduce the following restriction on permutations to avoid this kind of proof creation within equivalent proofs.

Definition 1 Two proofs $\mathcal{D}$ and $\mathcal{E} \vdash \Gamma$ are iso-initial, written $\mathcal{D} \simeq \mathcal{E}$, if each can be rewritten to the other using local permutations and the set of initial sequents in both $\mathcal{D}$ and $\mathcal{E}$ are the same. The sets under consideration are of pairs of formula occurrences.

The additional restriction on the sets of initial sequents allows the deletion and creation of subproofs during permutation only when such proofs are without initial rules. 
For the $T$-free fragment of MALL, this restriction is trivial, as all permutations preserve the set of initial sequents. However, because $T$ can arbitrarily rewrite a branch of a proof, allowing all permutations with $T$ would identify too many proofs. This restriction is further motivated by the observation from unit-free multiplicative proof nets, where the axiom links (which correspond to the initial sequents) contain the essential dynamics of a proof. These dynamics should not be suppressed by trivial permutations. Note that because we don't allow all permutations of $T$, we are decidedly not equating all proofs that are equated in the standard categorical model of MALL proofs; i.e., $\top$ is no longer a terminal object in a suitable $\star$-autonomous category where $\&$ is the Cartesian product.

\section{Multi-focusing for MALL}

In the remainder of this paper, we shall consider only cut-free proofs.

The formulas of MALL can be classified, based on their permutative affinities or polarity, into the following two classes.

(positive) $\quad P, Q, \ldots::=a|A \otimes B| \mathbf{1}|A \oplus B| \mathbf{0}$

(negative) $\quad N, M, \ldots::=a^{\perp}|A \not \gamma B| \perp|A \& B| \mathrm{T}$

A logical rule that applies to a positive (resp. negative) formula will henceforth be called a positive (resp. negative) rule. If $r_{1}$ is a positive rule and $r_{2}$ is a negative rule, then $r_{1} / r_{2}$ is an instance of the local permutation class pos/neg; similarly for pos/pos, neg/neg, and neg/pos. All pos/pos and neg/neg permutations are valid. Furthermore, neg/pos permutations are also valid since the negative rules are invertible and, hence, may be applied arbitrarily early (reading bottom-up). From a proof-search perspective, the negative rules are, therefore, asynchronous since their application does not depend on the structure of the side contexts. The positive rules, on the other hand, are noninvertible and, therefore, synchronous: their application depends on the structure of the remaining context and the sequence of rules that have been applied lower in the proof.

Andreoli [2] presented a focused proof system (for all of first-order linear logic) in which proofs have two phases. When reading proofs from the conclusion to the premises, a focal phase begins by granting focus to a positive formula from the available positive formulas: this focus can be indicated explicitly in the sequents by writing them as $\vdash \Gamma \Downarrow A$ where $A$ is under focus. Once the focused formula becomes negative, i.e., the sequent is of the form $\vdash \Gamma \Downarrow N$, the focus is released and the search enters the negative (asynchronous) phase where the negative connectives are decomposed; this phase is indicated in sequents of the form $\vdash \Gamma \Uparrow \Delta$. This phase separation is complete for cut-free proofs, i.e., every provable sequent has a focused proof $[2,16]$.

In this paper, we generalize this usual focusing strategy further in the following way: when deciding to focus, we may focus on more than one positive formula at a time, i.e., our positive sequents are now of the form $\vdash \Gamma \Downarrow \Delta$ (with $\Delta$ non-empty). All the formulas under focus are decomposed until only negative formulas remain in focus; 


$$
\begin{aligned}
& \frac{\vdash \Gamma_{1} \Downarrow A, \Delta_{1} \quad \vdash \Gamma_{2} \Downarrow B, \Delta_{2}}{\vdash \Gamma_{1}, \Gamma_{2} \Downarrow A \otimes B, \Delta_{1}, \Delta_{2}}[\otimes] \quad \frac{\vdash \Gamma \Downarrow A_{i}, \Delta}{\vdash \cdot \Downarrow \mathbf{1}}[\mathbf{1}] \quad \frac{\vdash \Gamma \Downarrow A_{1} \oplus A_{2}, \Delta}{\vdash}\left[\oplus_{i}\right] \\
& \frac{\vdash \Gamma \Uparrow A, \Delta \vdash \Gamma \Uparrow B, \Delta}{\vdash \Gamma \Uparrow A \& B, \Delta}[\&] \frac{\vdash \Gamma \Uparrow \mathrm{T}, \Delta}{\vdash \top}[\mathrm{\top}] \frac{\vdash \Gamma \Uparrow A, B, \Delta}{\vdash \Gamma \Uparrow A \mathcal{X} B, \Delta}[\mathcal{P}] \frac{\vdash \Gamma \Uparrow \Delta}{\vdash \Gamma \Uparrow \perp, \Delta}[\perp] \\
& \left.\frac{1-a^{\perp} \Downarrow a}{\vdash \mathrm{I}}\right] \quad \frac{\vdash \Gamma \Downarrow \Delta}{\vdash \Gamma, \Delta \Uparrow \cdot}[\mathrm{MF}] \quad \frac{\vdash \Gamma, A \Uparrow \Delta}{\vdash \Gamma \Uparrow A, \Delta}[\mathrm{R} \Uparrow] \quad \frac{\vdash \Gamma \Uparrow \Delta}{\vdash \Gamma \Downarrow \Delta}[\mathrm{R} \Downarrow]
\end{aligned}
$$

Fig. 2 Multi-focusing sequent calculus, MF. The contexts on the left of $\Downarrow$ and $\Uparrow$ contain only positive formulas or negated atoms. In the $[\mathrm{MF}]$ rule, $\Delta$ contains at least one positive formula. In the $[\mathrm{R} \Uparrow]$ rule, $A$ is positive or a negated atom. In the $[\mathrm{R} \Downarrow]$ rule, $\Delta$ is all negative. In $\left[\oplus_{i}\right], i \in\{1,2\}$.

then, the focus is released and the negative formulas are decomposed in the negative phase. The rules of this calculus of multi-focused proofs are presented in Figure 2.

Definition 2 If $\mathcal{D} \vdash \Gamma \Uparrow \Delta$ or $\mathcal{D} \vdash \Gamma \Downarrow \Delta$, then we write $\lfloor\mathcal{D}\rfloor$ for that proof of $\vdash \Gamma$, $\Delta$ that replaces every sequent of the form $\vdash \Gamma^{\prime} \Uparrow \Delta^{\prime}$ or $\vdash \Gamma^{\prime} \Downarrow \Delta^{\prime}$ in $\mathcal{D}$ with $\vdash \Gamma^{\prime}, \Delta^{\prime}$, elides all instances of $[\mathrm{R} \Uparrow],[\mathrm{R} \Downarrow]$ and $[\mathrm{MF}]$, and renames all other rules to their unbracketed forms $([\otimes]$ to $\otimes$, etc $)$.

\section{Theorem 3 (Correctness of multi-focusing)}

1. If $\mathcal{D} \vdash \Gamma \Downarrow \Delta$ or if $\mathcal{D} \vdash \Gamma \Uparrow \Delta$, then $\lfloor\mathcal{D}\rfloor \vdash \Gamma, \Delta$ (soundness).

2. If $\vdash \Gamma$, then $\vdash \cdot \Uparrow \Gamma$ (completeness).

Proof. Soundness is immediate. Completeness follows by observing that Andreoli's focusing calculus for MALL is recovered in MF by restricting the context $\Delta$ in $[\mathrm{MF}]$ to a singleton, and then using the analogous completeness theorem there $[2,16]$. Note that the proof in [2] is for full first-order, multiplicative-additive-exponential linear logic.

Given the phase separation induced by focusing, we define the following primitive equivalence on proofs that identifies proofs that differ from each other only inside a phase.

Definition 4 Two proofs $\mathcal{D}$ and $\mathcal{D}^{\prime} \vdash \Gamma \mathbb{} \Delta$ are iso-polar, written $\mathcal{D} \approx \mathcal{D}^{\prime}$, if they are equal up to permutations restricted to the pos/pos and neg/neg types.

This equivalence seems natural because the interchange of the pos/pos and neg/neg inference rules are truly parallel and non-interacting. Indeed, two iso-polar proofs have the same synthetic inference rules, i.e., the derived rules where the details of the positive and negative phases are elided, and only [I] and the phase transitions [R $\Downarrow]$ and $[\mathrm{MF}]$ are noted. For example, one proof of $\vdash a^{\perp}, a \otimes(b \& c), d \oplus \top \Uparrow \cdot$ using only synthetic rules is:

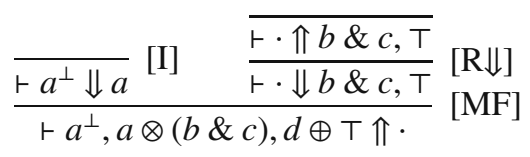


The instance of [MF] focuses on $a \otimes(b \& c)$ and $d \oplus T$, but the instances of $[\otimes]$ and

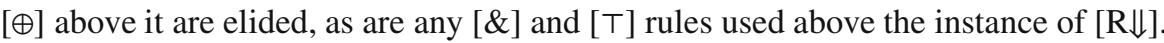

A single representative of the $\approx$-classes can be constructed by treating the contexts $\Delta$ to the right of $\Uparrow$ and $\Downarrow$ in MF as ordered contexts, similar to Andreoli's original focusing proof system [2]. This order on the context induces a fixed but arbitrary order of the pos/pos and neg/neg rules.

\section{Maximality and canonicity}

We now revisit the question of permutations of the synthetic inference rules induced by focusing. In the unfocused calculus, it is easy to see that the synthetic rule for a negative synthetic connective, which is a sequence of negative rules for the constituents of the synthetic connective, permutes with that of another synthetic negative connective: it is a simple matter of sequencing permutations. Similarly, the positive synthetic rules commute with other positive synthetic rules, and likewise for a neg/pos permutation of synthetic rules. As before, the only disallowed permutations in general are the pos/neg permutations.

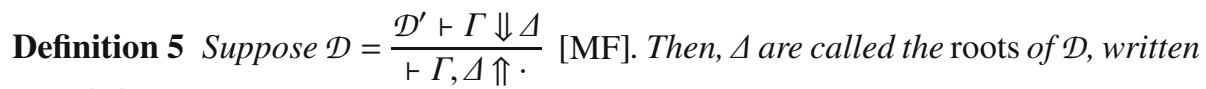
$\operatorname{roots}(\mathcal{D})$.

We intend to show that every member of an iso-initial class of proofs of $\vdash \Gamma$ is equivalent to a unique proof (upto iso-polarity) of $\vdash \cdot \Uparrow \Gamma$. In fact, we shall call these representatives of the iso-initial equivalence class the maximally multi-focused proofs.

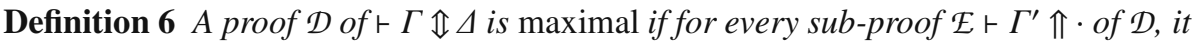
is the case for any $\mathcal{E}^{\prime} \simeq \mathcal{E} \vdash \Gamma^{\prime} \Uparrow \cdot$ that $\operatorname{roots}\left(\mathcal{E}^{\prime}\right) \subseteq \operatorname{roots}(\mathcal{E})$.

Our goal with maximal proofs is the following canonicity result:

Theorem 7 (canonicity) If $\mathcal{D} \simeq \mathcal{E} \vdash \Gamma \Uparrow \cdot$ are both maximal, then $\mathcal{D} \approx \mathcal{E}$.

The proof of this theorem will require considering permutations of entire synthetic connectives. Following Andreoli [2], we call a neighbouring pair of phases, with the bottom phase having a positive synthetic connective as its principal formula, and the top phase being its corresponding negative synthetic rules, a bipole. Consider two neighbouring bipoles: if the positive phase of the top bipole permutes with the negative phase of the bottom bipole, then in an unfocused form we can perform the permutation and merge the two bipoles by uniting their positive and negative phases, obtaining another (multi-)focused proof.

The MF rules are, however, too rigid to express any but the final points of the permutation. Thus, in this section we shall consider a comparitively more relaxed focusing calculus where a negative phase (of the bottom bipole) can be "carried through" the positive phase (of the top bipole). The bottom negative phase is first (temporarily) preempted by the top positive phase; for this, we use sequents of the form $\vdash \Gamma \downarrow \Delta ; \Xi$ 


$$
\begin{aligned}
& \frac{\vdash \Gamma_{1} \downarrow A, \Delta_{1} ; \Xi_{1} \quad \vdash \Gamma_{2} \downarrow B, \Delta_{2} ; \Xi_{2}}{\vdash \Gamma_{1}, \Gamma_{2} \downarrow A \otimes B, \Delta_{1}, \Delta_{2} ; \Xi_{1}, \Xi_{2}}[\otimes] \quad \frac{\vdash \Gamma \downarrow A_{i}, \Delta ; \Xi}{\vdash \cdot \downarrow \mathbf{1} ; \cdot}[\mathbf{1}] \quad \frac{\vdash \Gamma \downarrow A_{1} \oplus A_{2}, \Delta ; \Xi}{\left.\vdash \oplus_{i}\right]}
\end{aligned}
$$

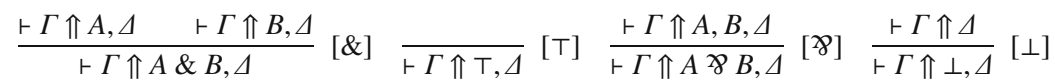

$$
\begin{aligned}
& \frac{\vdash a^{\perp} \downarrow a ; \cdot}{\vdash \mathrm{I}]} \frac{\vdash \Gamma \downarrow \Delta ; \Xi}{\vdash \Gamma, \Delta \Uparrow \Xi}\left[\mathrm{PMF}_{1}\right] \frac{\vdash \Gamma \downarrow \Delta, \Psi ; \Xi}{\vdash \Gamma, \Delta \downarrow \Psi ; \Xi}\left[\mathrm{PMF}_{2}\right] \\
& \frac{\vdash \Gamma, A \Uparrow \Delta}{\vdash \Gamma \Uparrow A, \Delta}[\mathrm{R} \Uparrow] \frac{\vdash \Gamma \downarrow \Delta ; N, \Xi}{\vdash \Gamma \downarrow \Delta, N ; \Xi}[\mathrm{R} \downarrow] \frac{\vdash \Gamma \Uparrow \Xi}{\vdash \Gamma \downarrow \cdot ; \Xi}[\mathrm{R}]
\end{aligned}
$$

Fig. 3 Rules of the pre-emptive multi-focusing calculus, PMF. All side conditions from MF (Fig. 2) are carried over; in particular, for $\left[\mathrm{PMF}_{1}\right]$ and $\left[\mathrm{PMF}_{2}\right]$, the context $\Delta$ is non-empty.

where $\Delta$ is under focus, and $\Xi$ is a suspended context. Later, when the positive phase has permuted down, the negative phases are awakened into active sequents of the form $\vdash \Gamma \Uparrow \Delta$. The rules of this pre-emptive multi-focusing calculus, called PMF, are in Figure 3. A straightforward injection (-) $)^{\#}$ from MF to PMF derivations is assumed.

Fact 8 The following are seen by straightforward induction.

1. If $\vdash_{\mathrm{MF}} \Gamma \Downarrow \Delta$, then $\vdash_{\mathrm{PMF}} \Gamma \downarrow \Delta ; \cdot$

2. If $\vdash_{\mathrm{PMF}} \Gamma \downarrow \Delta ; \Xi$, then $\vdash_{\mathrm{MF}} \Gamma \Downarrow \Delta, \Xi$.

3. $\vdash_{\mathrm{MF}} \Gamma \Uparrow \Delta$ if and only if $\vdash_{\mathrm{PMF}} \Gamma \Uparrow \Delta$.

Because both positive and negative phases can be pre-empted using the $\left[\mathrm{PMF}_{i}\right]$ rules, we can explicitly sequence two positive phases by introducing new instances of $\left[\mathrm{PMF}_{2}\right]$. Note that focus, once granted, cannot be removed until the formula becomes negative; thus, PMF does not destroy synthetic positive connectives, which are the essential innovation of focusing. After the positive phase of the top bipole has permuted through the negative phase of the bottom bipole, the suspended negative phases are awakened, which might give rise to a number of different sub-derivations (due to $\&$ ). If $\mathbf{D}$ is this multiset of sub-derivations, then we indicate that it finishes with the negative phase for $\Xi$ as $\mathbf{D} / \Xi$.

\section{Definition 9}

1. (D $/ \Xi) \vdash \Gamma \downarrow \Delta ; \Xi$, where $\mathbf{D}$ is a multiset of derivations, has one of the following forms:

$$
\begin{aligned}
& \frac{(\mathbf{D} / N, \Xi) \vdash \Gamma \downarrow \Delta ; N, \Xi}{\vdash \Gamma \downarrow \Delta, N ; \Xi}[\mathrm{R} \downarrow] \quad \frac{(\mathbf{D} / \Xi) \vdash \Gamma \Uparrow \Xi}{\vdash \Gamma \downarrow \cdot \Xi}[\mathrm{R}] \frac{(\mathbf{D} / \Xi) \vdash \Gamma \downarrow \Delta, A_{i} ; \Xi}{\vdash \Gamma \downarrow \Delta, A_{1} \oplus A_{2} ; \Xi}\left[\oplus_{i}\right] \\
& \frac{(\mathbf{D} / \Xi) \vdash \Gamma_{1} \downarrow \Delta_{1}, A ; \Xi \quad \mathcal{E} \vdash \Gamma_{2} \downarrow \Delta_{2}, B ; \cdot}{\vdash \Gamma_{1}, \Gamma_{2} \downarrow \Delta_{1}, \Delta_{2}, A \otimes B ; \Xi}[\otimes] \frac{(\mathbf{D} / \Xi) \vdash \Gamma \downarrow \Delta, \Delta^{\prime} ; \Xi}{\vdash \Gamma, \Delta^{\prime} \downarrow \Delta ; \Xi}\left[\mathrm{PMF}_{2}\right]
\end{aligned}
$$

(And the symmetric case for $[\otimes]$.)

2. $(\mathbf{D} / \Xi) \vdash \Gamma \Uparrow \Delta, \Xi$ where $\mathbf{D}$ is a multiset of derivations, has one of the following forms: 


$$
\begin{aligned}
& \frac{\left(\mathbf{D}_{1} / \Xi^{\prime}, A\right) \vdash \Gamma \Uparrow \Delta, \Xi^{\prime}, A \quad\left(\mathbf{D}_{2} / \Xi^{\prime}, B\right) \vdash \Gamma \Uparrow \Delta, \Xi^{\prime}, B}{\vdash \Gamma \Uparrow \Delta, \Xi^{\prime}, A \& B}[\&] \quad \ldots \text { and } \mathbf{D}=\mathbf{D}_{1}, \mathbf{D}_{2} \\
& \overline{\vdash \Gamma \Uparrow \Delta, \Xi^{\prime}, \top}[\top] \quad \ldots \text { and } \mathbf{D}=\text {. } \\
& \frac{\left(\mathbf{D} / \Xi^{\prime}, A, B\right) \vdash \Gamma \Uparrow \Delta, \Xi^{\prime}, A, B}{\vdash \Gamma \Uparrow \Delta, \Xi^{\prime}, A \mathcal{P} B}[\mathcal{P}] \frac{\left(\mathbf{D} / \Xi^{\prime}\right) \vdash \Gamma \Uparrow \Delta, \Xi^{\prime}}{\vdash \Gamma \Uparrow \Delta, \Xi^{\prime}, \perp}[\perp] \\
& \frac{\left(\mathbf{D} / \Xi^{\prime}\right) \vdash \Gamma, P \Uparrow \Delta, \Xi^{\prime}}{\vdash \Gamma \Uparrow \Delta, \Xi^{\prime}, P}[\mathrm{R} \Uparrow]
\end{aligned}
$$

with $\Xi=\Xi^{\prime}, F$ for $F$ being $A \not \mathcal{B}, A \& B, \top, \perp$, or $P$. Additionally, $(\mathcal{D} / \cdot)=\mathcal{D}$.

We define the merge operation in terms of a rewrite $\longrightarrow$ between PMF proofs such that in each case of the rewrite at least one root of a $\left[\mathrm{PMF}_{1}\right]$ is permuted lower in the derivation. Eventually, this will bring two instances of $\left[\mathrm{PMF}_{i}\right]$ next to each other, at which point they are merged. All negative rules encountered during the rewrite are immediately suspended, causing them to permute above the positive phase rooted at the $\left[\mathrm{PMF}_{i}\right]$ being permuted. To obtain confluence globally, we must first split the roots to obtain the subset that can merge with the roots of the bottom bipole; otherwise, we might merge bipoles in the wrong order and block possible merges.

Definition 10 The rewrite $\longrightarrow$ between PMF proofs has the following rules.

$$
\begin{aligned}
& \frac{\mathcal{D} \vdash \Gamma \downarrow \Delta, \Delta^{\prime} ; \Xi}{\vdash \Gamma, \Delta, \Delta^{\prime} \Uparrow \Xi}\left[\mathrm{PMF}_{1}\right] \quad \longrightarrow \quad \frac{\frac{\mathcal{D} \vdash \Gamma \downarrow \Delta, \Delta^{\prime} ; \Xi}{\vdash \Gamma, \Delta \downarrow \Delta^{\prime} ; \Xi}\left[\mathrm{PMF}_{2}\right]}{\vdash \Gamma, \Delta, \Delta^{\prime} \Uparrow \Xi}\left[\mathrm{PMF}_{1}\right] \\
& \frac{(\mathbf{D} / \Xi) \vdash \Gamma, P \downarrow \Delta ; \Xi}{\frac{\vdash \Gamma, P, \Delta \Uparrow \Xi}{\vdash \Gamma, \Delta \Uparrow \Xi, P}[\mathrm{R} \Uparrow]}\left[\mathrm{PMF}_{1}\right] \quad \longrightarrow \quad \frac{(\mathbf{D} / \Xi, P) \vdash \Gamma \downarrow \Delta ; P, \Xi}{\vdash \Gamma, \Delta \Uparrow \Xi, P}\left[\mathrm{PMF}_{1}\right]
\end{aligned}
$$

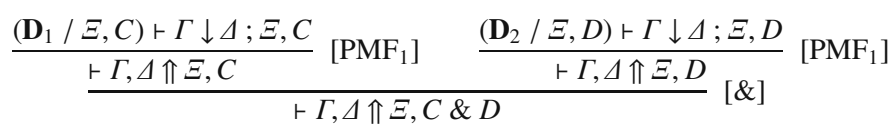

$$
\begin{aligned}
& \longrightarrow \frac{\left(\mathbf{D}_{1}, \mathbf{D}_{2} / \Xi, C \& D\right) \vdash \Gamma \downarrow \Delta ; \Xi, C \& D}{\vdash \Gamma, \Delta \Uparrow \Xi, C \& D}\left[\mathrm{PMF}_{1}\right] \\
& \frac{(\mathbf{D} / \Xi, C, D) \vdash \Gamma \downarrow \Delta ; \Xi, C, D}{\frac{\vdash \Gamma, \Delta \Uparrow \Xi, C, D}{\vdash \Gamma, \Delta \Uparrow \Xi, C \ngtr D}\left[\mathrm{PMF}_{1}\right]} \rightarrow \frac{(\mathbf{D} / \Xi, C \ngtr D) \vdash \Gamma \downarrow \Delta ; \Xi, C \ngtr D}{\vdash \Gamma, \Delta \Uparrow \Xi, C \ngtr D}\left[\mathrm{PMF}_{1}\right] \\
& \frac{(\mathbf{D} / \Xi) \vdash \Gamma \downarrow \Delta ; \Xi}{\frac{\vdash \Gamma, \Delta \Uparrow \Xi}{\vdash \Gamma, \Delta \Uparrow \Xi, \perp}[\perp]}\left[\mathrm{PMF}_{1}\right] \quad \longrightarrow \frac{(\mathbf{D} / \Xi, \perp)+\Gamma \downarrow \Delta ; \Xi, \perp}{\vdash \Gamma, \Delta \Uparrow \Xi, \perp}\left[\mathrm{PMF}_{1}\right] \\
& \frac{(\mathbf{D} / N, \Xi) \vdash \Gamma \downarrow \Delta, \Psi ; N, \Xi}{\frac{\vdash \Gamma, \Delta \downarrow \Psi ; N, \Xi}{\vdash \Gamma, \Delta \downarrow \Psi, N ; \Xi}[\mathrm{R} \downarrow]}\left[\mathrm{PMF}_{1}\right] \quad \longrightarrow \quad \frac{(\mathbf{D} / N, \Xi) \vdash \Gamma \downarrow \Delta, \Psi ; N, \Xi}{\frac{\vdash \Gamma \downarrow \Delta, \Psi, N ; \Xi}{\vdash \Gamma, \Delta \downarrow \Psi, N ; \Xi}\left[\mathrm{PMF}_{1}\right]}[\mathrm{R} \downarrow] \\
& \frac{(\mathbf{D} / \Xi)+\Gamma \downarrow \Delta ; \Xi}{\frac{\vdash \Gamma, \Delta \Uparrow \Xi}{\vdash \Gamma, \Delta \downarrow \cdot ; \Xi}[\mathrm{R}]}\left[\mathrm{PMF}_{1}\right] \quad \longrightarrow \quad \frac{(\mathbf{D} / \Xi) \vdash \Gamma \downarrow \Delta ; \Xi}{\vdash \Gamma, \Delta \downarrow \cdot ; \Xi}\left[\mathrm{PMF}_{2}\right]
\end{aligned}
$$




$$
\begin{aligned}
& \frac{\frac{\mathcal{D} \vdash \Gamma_{1} \downarrow \Psi, \Delta_{1}, A ; \Xi_{1}}{\vdash \Gamma_{1}, \Psi \downarrow \Delta_{1}, A ; \Xi_{1}}\left[\mathrm{PMF}_{2}\right] \quad \mathcal{E} \vdash \Gamma_{1} \downarrow \Delta_{2}, B ; \Xi_{2}}{\vdash \Gamma_{1}, \Gamma_{2}, \Psi \downarrow \Delta_{1}, \Delta_{2}, A \otimes B ; \Xi_{1}, \Xi_{2}}[\otimes] \\
& \longrightarrow \quad \frac{\mathcal{D} \vdash \Gamma_{1} \downarrow \Psi, \Delta_{1}, A ; \Xi_{1} \quad \mathcal{E} \vdash \Gamma_{1} \downarrow \Delta_{2}, B ; \Xi_{2}}{\frac{\vdash \Gamma_{1}, \Gamma_{2} \downarrow \Psi, \Delta_{1}, \Delta_{2}, A \otimes B ; \Xi_{1}, \Xi_{2}}{\vdash \Gamma_{1}, \Gamma_{2}, \Psi \downarrow \Delta_{1}, \Delta_{2}, A \otimes B ; \Xi_{1}, \Xi_{2}}\left[\mathrm{PMF}_{2}\right]}[\otimes] \\
& \frac{\frac{\mathcal{D} \vdash \Gamma \downarrow \Psi, \Delta, A_{i} ; \Xi}{\vdash \Gamma, \Psi \downarrow \Delta, A_{i} ; \Xi}\left[\mathrm{PMF}_{2}\right]}{\vdash \Gamma, \Psi \downarrow \Delta, A_{1} \oplus A_{2} ; \Xi}\left[\oplus_{i}\right] \quad \longrightarrow \quad \frac{\frac{\mathcal{D} \vdash \Gamma \downarrow \Psi, \Delta, A_{i} ; \Xi}{\vdash \Gamma \downarrow \Psi, \Delta, A_{1} \oplus A_{2} ; \Xi}\left[\oplus_{i}\right]}{\vdash \Gamma, \Psi \downarrow \Delta, A_{1} \oplus A_{2} ; \Xi}\left[\mathrm{PMF}_{2}\right] \\
& \frac{\mathcal{D} \vdash \Gamma \downarrow \Psi_{1}, \Psi_{2}, \Delta ; \Xi}{\vdash \Gamma, \Psi_{1} \downarrow \Delta, \Psi_{2} ; \Xi}\left[\mathrm{PMF}_{2}\right] \quad \longrightarrow \quad \frac{\mathcal{D} \vdash \Gamma \downarrow \Psi_{1}, \Psi_{2}, \Delta ; \Xi}{\vdash \Gamma, \Psi_{1}, \Psi_{2} \downarrow \Delta ; \Xi}\left[\mathrm{PMF}_{2}\right] \\
& \frac{\mathcal{D} \vdash \Gamma \downarrow \Psi_{1}, \Psi_{2}, \Delta ; \Xi}{\frac{\vdash \Gamma, \Psi_{1} \downarrow \Psi_{2} ; \Xi}{\vdash \Gamma, \Psi_{1}, \Psi_{2} \Uparrow \Xi}\left[\mathrm{PMF}_{2}\right]} \quad \longrightarrow \quad \frac{\mathcal{D} \vdash \Gamma \downarrow \Psi_{1}, \Psi_{2} ; \Xi}{\vdash \Gamma, \Psi_{1}, \Psi_{2} \Uparrow \Xi}\left[\mathrm{PMF}_{1}\right]
\end{aligned}
$$

The symmetric cases for $\left[\mathrm{PMF}_{1}\right] /[\otimes]$ and $\left[\mathrm{PMF}_{1}\right] /[\oplus]_{i}$ are elided.

The rewrite in defn. 10 is a permutation on MF derivations modulo the injection into PMF. The intermediate points of the permutation after the injection are not interesting, but the reflexive-transitive closure of the PMF rewrite also defines the following MF rewrite.

Definition 11 If $\mathcal{D}, \mathcal{E} \vdash_{\mathrm{MF}} \Gamma \Uparrow \Delta$, and $\mathcal{D}^{\#} \longrightarrow^{*} \mathcal{E}^{\#}$, then $\mathcal{D} \longrightarrow \mathcal{E}$.

We shall show that this rewrite on MF derivations will generate the maximal proofs. The proof itself will be a trivial consequence of two decomposition lemmas. The leftdecomposition lemma below shows that the maximal proofs are $\longrightarrow$-normal upto isopolarity.

\section{Lemma 12 (left decomposition)}

If $\mathcal{D} \vdash \Gamma \mathbb{1} \Delta$ is maximal and $\mathcal{D} \longrightarrow \mathcal{E}$, then $\mathcal{D} \approx \mathcal{E}$.

Proof. Note that in every case of the rewrite $\longrightarrow$ on PMF derivations, an instance of $\left[\mathrm{PMF}_{1}\right]$ is brought closer to the root of the derivation. Therefore, the rewrite $\longrightarrow$ on MF proofs can only enlarge the lowermost roots in $\mathcal{D}$. But, $\mathcal{D}$ is already maximal. So $\mathcal{E}$ has the same instances of $[\mathrm{MF}]$ as $\mathcal{D}$, i.e., $\mathcal{D} \approx \mathcal{E}$.

The second key lemma is a right-decomposition that establishes that the maximal proofs are reachable by $\longrightarrow$.

\section{Lemma 13 (right decomposition)}

If $\mathcal{D} \simeq \mathcal{E} \vdash_{\mathrm{MF}} \Gamma \mathbb{} \Delta$ and $\mathcal{E}$ is maximal, then $\mathcal{D} \longrightarrow \mathcal{E}$.

Proof (Sketch). We have to show that all ways of permuting a root downwards in a proof can be generated by $\longrightarrow$. But this is easily seen because the $\longrightarrow$ is allowed to divide the roots and permute only the necessary fragment downwards. For a representative example, suppose the following is a sub-derivation of $\mathcal{D}^{\#}$ : 


$$
\mathcal{F}=\frac{\frac{\mathcal{F}^{\prime} \vdash \Gamma, P \downarrow \Delta, Q ; \cdot}{\frac{\vdash \Gamma, P, Q, \Delta \Uparrow \cdot}{\vdash \Gamma, \Delta \Uparrow P, Q}\left[\mathrm{PMF}_{1}\right]}}{\frac{\mathrm{R} \Uparrow]^{2}}{\vdash \Gamma, \Delta \Uparrow P \mathcal{P} Q}[\mathcal{P}]}
$$

Of the roots $\Delta, Q$, only $\Delta$ can possibly permute below $P \& Q$, because $Q$ is one of its sub-formulas. According to the rewrite rules, we first remove $Q$ from the roots of the $[\mathrm{PMF}]$ rule by inserting another $[\mathrm{PMF}]$. The permutation can now proceed (for some $\left.\mathbf{F} / P, Q \simeq \mathcal{F}^{\prime \prime}\right)$ :

$$
\begin{aligned}
& \mathcal{F}^{\prime \prime}=\frac{\mathcal{F}^{\prime} \vdash \Gamma, P \downarrow \Delta, Q ; \cdot}{\vdash \Gamma, P, Q \downarrow \Delta ; \cdot}\left[\mathrm{PMF}_{2}\right]
\end{aligned}
$$

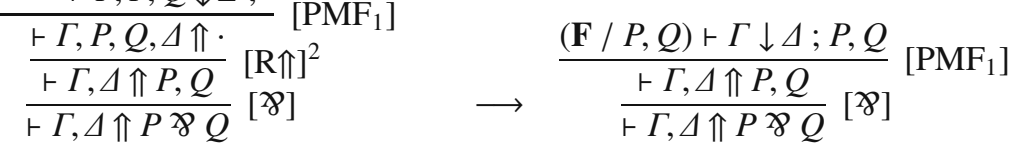

$$
\begin{aligned}
& \longrightarrow \frac{(\mathbf{F} / P \mathcal{P} Q) \vdash \Gamma \downarrow \Delta ; P \not \mathcal{Q} Q}{\vdash \Gamma, \Delta \Uparrow P \mathcal{P} Q}\left[\mathrm{PMF}_{1}\right]
\end{aligned}
$$

The instance of $\left[\mathrm{PMF}_{1}\right]$ that permutes down is free of the disallowed root $Q$.

Proof (of theorem 7). Let $\mathcal{D} \simeq \mathcal{E} \vdash_{\mathrm{MF}} \Gamma \mathbb{} \Delta$ be given such that both $\mathcal{D}$ and $\mathcal{E}$ are maximal. By lemma $13, \mathcal{D} \longrightarrow \mathcal{E}$; hence, by lemma $12, \mathcal{D} \approx \mathcal{E}$.

\section{Multi-focusing and proof nets}

The usual approach to the proof identity problem in linear logic (and to providing a canonical representation of proofs) consists in using proof nets which were first introduced by Girard [9]. Since we proved that maximally multi-focused proofs also provide such a canonical approach to proofs it is natural to compare our approach with proof nets. This is the aim of the present section where we deal with a restricted fragment of MALL proofs, the unit-free cut-free multiplicative fragment, $\mathrm{MLL}^{-}$, for which proof nets are especially well-behaved: we shall provide a direct proof that maximally multi-focused proofs in $\mathrm{MLL}^{-}$are in a one-to-one correspondence with cut-free $\mathrm{MLL}^{-}$ proof nets.

The previous results of the paper already ensure that such a result is true but we shall now give a direct evidence of this fact by actually building the class of iso-polar maximally multi-focused proofs corresponding to a given proof net. The converse, namely that two iso-polar maximally multi-focused proofs correspond to the same proof nets is trivial.

Proof nets are structures that do not retain all the unnecessary ordering information contained in a sequent proof. A $\mathrm{MLL}^{-}$proof structure is thus a graph structure consisting in the formula tree of the sequent $\vdash \Gamma$ together with some more structure representing the initial rules: 

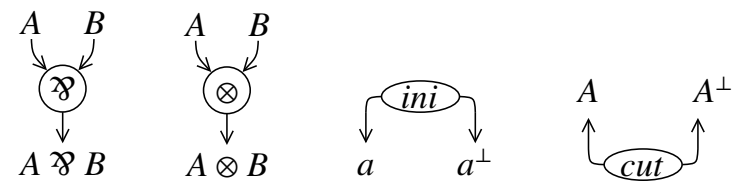

Fig. 4 Unit-free cut-free MLL proof nets. ini is restricted to the atomic formulas.

Definition 14 (MLL- proof structure) $A M L L^{-}$proof structure on $\vdash \Gamma$ is a graph made of cells represented in Figure 4 which are linked by edges labeled with $M L^{-}$ formulas. There is one pending edge for each formula $F$ in $\vdash \Gamma$ which is labeled with $F$ and which is called a conclusion.

Additional conditions are imposed in order to ensure that this proof structure is actually a logical object and represents a proof:

Definition 15 (MLL- proof net) $A M L L^{-}$proof net on $\vdash \Gamma$ is a proof structure that results from the desequentialization of a sequent proof $\pi$ of $\vdash \Gamma$ by forgetting the inference rule ordering ${ }^{1}$.

The previous definition does not provide a convenient criterion that can be helpful to check that a given proof structure is indeed a proof net. Many more satisfying criteria have been provided to characterize proof nets, they all have in common not to be inductive but geometric criteria (they deal with the structure as a whole, not as made of elementary components). In the following, we shall only consider cut-free $\mathrm{MLL}^{-}$ proof structures.

As already mentioned, we shall now be interested in providing a direct proof of the following theorem:

Theorem 16 Two maximally multi-focused $M L L^{-}$proofs of $\vdash \cdot \Uparrow \Gamma$ are iso-polar iff they have the same $M L L^{-}$proof net.

The theorem will be proved by showing that for every proof net there is a unique maximally multi-focused proof (up to iso-polarity) associated with it. We first recall two definitions from [1] which develops a focused sequentialization algorithm for $\mathrm{MLL}^{-}$proof nets:

Definition $17\left(\operatorname{split}(\pi)\right.$, foc $(\pi)$, from [1]) Let $\pi$ be an $M L L^{-}$proof net.

1. $\operatorname{split}(\pi)$ is the set of positive conclusions $P$ of $\pi$ such that removing the concluding $\otimes$-link of $P$ disconnects $\pi$ in two proof nets $\pi_{1}$ and $\pi_{2}$.

2. $\operatorname{foc}(\pi)$ is the set of conclusions $F$ of $\pi$ such that $F$ is a positive atom and $\pi$ is just an ini link; or $F \in \operatorname{split}(\pi)$ and its premisses $A$ and $B$ are conclusions of the two sub-nets $\pi_{1}$ and $\pi_{2}$ where $A$ (resp. B) is negative or $A \in \operatorname{foc}\left(\pi_{1}\right)$ (resp. $\left.B \in \operatorname{foc}\left(\pi_{2}\right)\right)$.

\footnotetext{
${ }^{1}$ A MLL $^{-}$inference rule is turned to the corresponding cell of Figure 4 and the cells are combined by tracing the formulas occurrences in the sequent proof.
} 
Proof (of Theorem 16). Let $\pi$ be a $\mathrm{MLL}^{-}$proof net of conclusions $\Gamma$. We outline a sequentialization algorithm producing a maximally multi-focused proof of conclusion $\vdash \cdot \Uparrow \Gamma$ if $\Gamma$ contains some negative non-atomic formula or $\vdash \Gamma \backslash \operatorname{foc}(\pi) \Downarrow \operatorname{foc}(\pi)$ otherwise. We reason by induction on the size of $\pi$.

Case $\Gamma$ contains at least one negative formula. We remove all negative cells (that is, the $\gamma$ cells) of $\pi$ up to reaching a positive cell or an initial cell. The resulting proof structure is a proof net $\pi^{\prime}$ and its conclusions $\Gamma^{\prime}$ are positive. By induction hypothesis, we can sequentialize it into a maximally multi-focused proof $\mathcal{D}^{\prime}$ of conclusion $\vdash \Gamma^{\prime} \backslash$ foc $\left(\pi^{\prime}\right) \Downarrow \operatorname{foc}\left(\pi^{\prime}\right)$ by sequentializing in an arbitrary order (the different possibilities give rise to iso-polar proofs) the negative rules that have been removed in the previous step, we obtain a proof $\mathcal{D}$ of the form:

$$
\frac{\mathcal{D}^{\prime} \vdash \Gamma^{\prime} \backslash \operatorname{foc}\left(\pi^{\prime}\right) \Downarrow \text { foc }\left(\pi^{\prime}\right)}{\frac{\vdash \Gamma^{\prime} \Uparrow \cdot}{\vdots}[\mathrm{MF}]}
$$

Case $\Gamma$ contains only positive formulas. Since $\pi$ is a proof net, $\operatorname{foc}(\pi) \neq \emptyset$. Consider the formulas in $[(] \pi)$ and remove the top-most positive connectives of every $F \in \operatorname{foc}(\pi)$. The resulting proof structure is not a proof net since it is not connected; however, each of its connected components is. Let them be $\pi_{1}, \ldots, \pi_{n}$. For $1 \leq i \leq n, \pi_{i}$ has conclusions $\Gamma_{i}$ which has at least one negative formula or which is reduced to an axiom link. In the first case, one can inductively sequentialize it into of maximally multi-focused proof $\mathcal{D}_{i}$. In order to conclude, we only need to show that one can obtain a proof of $\vdash \cdot \Uparrow \Gamma$ from the $\mathcal{D}_{i}$ and the positive cells of the formulas of $\operatorname{foc}(\pi)$, which follows from the fact that the formulas in $\operatorname{foc}(\pi)$ are hereditarily splitting: applying these formulas in any order (as long as the sub-formula priority is maintained), gives rise to a way to sequentialize $\pi$.

We finally need to check that the proof obtained with this process is indeed maximal, but this is done very easily: let $F$ be a formula that could potentially enlarge the set of foci and let us consider a proof $\mathcal{D}_{F}$ that witnesses this fact ( $\mathcal{D}_{F}$ focuses on $F$ ). By desequentializing $\mathcal{D}_{F}$, we get a proof net $\pi$ and since $\mathcal{D}_{F}$ is a sequentialization of $\pi$ that focuses on $F$ which is positive, then $F$ is hereditarily splitting, that is $F \in \operatorname{foc}(\pi)$, so $\operatorname{foc}(\pi)$ is maximal.

The process considered in this proof is non-deterministic (within a negative or positive phase, we sequentialize in any order) and we can check that the different proofs that can result from this process are exactly all the iso-polar maximally multi-focused proofs of the iso-polarity class corresponding to proof net $\pi$.

We showed in this section that there is a bijection between $\mathrm{MLL}^{-}$proof nets and classes of iso-polar maximally multi-focused proofs. $\mathrm{MLL}^{-}$proof nets are certainly the most concise canonical structures for this fragment. There are candidates to extend $\mathrm{MLL}^{-}$proof nets to broader fragments (MLL with units [14], MALL [12] or MELL) but they are not as satisfactory as for $\mathrm{MLL}^{-}$. The analysis we just made could be 
carried to MALL proof nets as introduced by Hughes and van Glabbeek [12] for the appropriate extension of definition 17 (in particular to take into account the fact that with MALL proof nets there is not only one linking but a set of linkings corresponding to the additive slices of the proof net).

The problem of proof-nets for MALL with units is still open. Yet, these fragments have standard sequent calculi with well understood focusing systems. We expect that an analysis of the maximally multi-focused sequent proofs would yield a better understanding of proof net-like structures for such fragments.

\section{Conclusion}

The contributions of this paper are three-fold: (i) we extend Andreoli's definition of focused proofs to multi-focused proofs, for which we define a notion of maximality; (ii) we show that the maximally multi-focused proofs are representatives of their $\simeq$ equivalence class upto a trivial iso-polar equivalence; and (iii) we prove that unit-free multiplicative proof nets are in bijective correspondence with maximal multi-focused proofs for unit-free multiplicative linear logic.

The notion of multi-focusing in this paper was first considered by Saurin and Miller [16] as naturally arising in the structure of focalization graphs to prove the focalization theorem. Multi-focusing was subsequently also used by Delande and Miller [7] as a necessary generalization of Andreoli's asymmetric treatment of the positive formulas. Andreoli studied focusing in proof nets [1, 3] and defined a notion of "multi-focus" [3] with a different meaning: there, it refers to a part of the context which is needed in order to apply the decide rule. He also investigates the use of focusing to construct proof nets for a restricted fragment of $\mathrm{MLL}^{-}$.

Faggian et al [8,6] introduced L-nets as a generalization of designs from Girard's ludics [11]: L-nets can be seen as designs with a flexible degree of sequentiality, falling between sequent proofs and proof nets. This appears similar to multi-focusing which covers the spectrum from singly focused proofs to maximally multi-focused proofs, and thus exhibits some flexibility about the degree of sequentiality. This flexibility is also observed in [7] which presents the search for proofs and refutations as a pair of mutually normalizing interpretations of a neutral procedure for the cut and atom-free MALL. Relating these diverse approaches is an important matter for future work.

Several other open questions remain about multi-focused proofs. Firstly, we lack a cut-elimination theorem for multi-focused proofs that generalizes similar theorems for singly focused proofs (see, eg. $[4,15]$ ). Moreover, it is considerably unclear how maximality interacts with cut-elimination, for the standard procedure would not preserve maximality. In terms of larger fragments of linear logic, multi-focusing generalizes easily to admit the exponentials and first-order quantification; however, the respective notions of maximality remain to be developed for these fragments. 


\section{References}

1. J.-M. Andreoli and R. Maieli. Focusing and proof nets in linear and noncommutative logic. In International Conference on Logic for Programming and Automated Reasoning (LPAR), volume 1581 of LNAI. Springer, 1999.

2. Jean-Marc Andreoli. Logic programming with focusing proofs in linear logic. J. of Logic and Computation, 2(3):297-347, 1992.

3. Jean-Marc Andreoli. Focussing proof-net construction as a middleware paradigm. In Andrei Voronkov, editor, 18th Conference on Automated Deduction (CADE), number 2392 in LNAI, pages 501-516. Springer, 2002.

4. Kaustuv Chaudhuri, Frank Pfenning, and Greg Price. A logical characterization of forward and backward chaining in the inverse method. J. of Automated Reasoning, 40(2-3):133-177, March 2008.

5. Pierre-Louis Curien. Introduction to linear logic and ludics, Part I. Advances in Mathematics (China), 34(5):513-544, January 2005.

6. Pierre-Louis Curien and Claudia Faggian. L-nets, strategies and proof-nets. In C.-H. Luke Ong, editor, CSL 2005: Computer Science Logic, volume 3634 of LNCS, pages 167-183. Springer, 2005.

7. Olivier Delande and Dale Miller. A neutral approach to proof and refutation in MALL. In F. Pfenning, editor, 23th Symp. on Logic in Computer Science. IEEE Computer Society Press, 2008.

8. Claudia Faggian and François Maurel. Ludics nets, a game model of concurrent interaction. In 20th Symp. on Logic in Computer Science, pages 376-385. IEEE Computer Society, 2005.

9. Jean-Yves Girard. Linear logic. Theoretical Computer Science, 50:1-102, 1987.

10. Jean-Yves Girard. Proof-nets: the parallel syntax for proof-theory. In Aldo Ursini and Paolo Agliano, editors, Logic and Algebra, volume 180 of Lecture Notes In Pure and Applied Mathematics, pages 97-124, New York, 1996. Marcel Dekker.

11. Jean-Yves Girard. Locus solum. Mathematical Structures in Computer Science, 11(3):301-506, June 2001.

12. Dominic Hughes and Rob Van Glabbeek. Proof nets for unit-free multiplicative-additive linear logic. ACM Trans. on Computational Logic, 6:784-842, 2005.

13. Stephen Cole Kleene. Permutabilities of inferences in Gentzen's calculi LK and LJ. Memoirs of the American Mathematical Society, 10:1-26, 1952.

14. François Lamarche and Lutz Straßburger. From proof nets to the free *-autonomous category. Logical Methods in Computer Science, 2(4:3):1-44, 2006.

15. Chuck Liang and Dale Miller. Focusing and polarization in intuitionistic logic. In J. Duparc and T. A. Henzinger, editors, CSL 2007: Computer Science Logic, volume 4646 of LNCS, pages 451-465. Springer, 2007.

16. Dale Miller and Alexis Saurin. From proofs to focused proofs: a modular proof of focalization in linear logic. In J. Duparc and T. A. Henzinger, editors, CSL 2007: Computer Science Logic, volume 4646 of $L N C S$, pages 405-419. Springer, 2007. 